\title{
The Future of Prostate Cancer Risk Prediction
}

\author{
Kenneth Ross Muir ${ }^{1,2}$ - Artitaya Lophatananon ${ }^{1,2}$ • \\ Vincent Gnanapragasam ${ }^{3}$. Jonathan Rees ${ }^{4}$
}

Published online: 28 September 2015

(C) Springer International Publishing AG 2015

\begin{abstract}
Prostate cancer (PCA) is a difficult disease to diagnose and treat appropriately as the majority of cases detected have an indolent form and do not have a major impact on mortality. The real challenge therefore is being able to selectively identify the aggressive forms of the disease and treat those effectively. "Risk" potentially offers a unifying concept for improving the diagnostic and care pathways for PCA. It also has the potential to inform men on their underlying risk to consider possible preventive and enhanced screening strategies. Risk prediction models can help guide both the patient and the medical team in deciding on the most appropriate diagnostic and treatment pathways to follow. This review will focus on current risk prediction models for diagnosis in the community and predict likely further trends for the wider developments of risk models to more fully inform the full prevention, diagnostic and treatment management options for PCA.
\end{abstract}

Keywords Prostate cancer - Risk prediction models · Nomograms $\cdot$ PSA $\cdot$ Diagnostic

This article is part of the Topical Collection on Cancer Epidemiology

Kenneth Ross Muir

kenneth.muir@manchester.ac.uk

1 Institute of Population Health, University of Manchester, Manchester, UK

2 Warwick Medical School, Warwick University, Coventry, UK

3 Academic Urology Group, Department of Surgery and Oncology, University of Cambridge, Cambridge, UK

4 General Practitioner Backwell and Nailsea Medical Group, Bristol, UK

\section{Introduction}

\section{Diagnostic Risk Calculators}

Historically, the diagnosis of prostate cancer (PCA) has been dominated by a single test, prostate-specific antigen (PSA) which when combined with age, clinical examination and medical history can allow for a prediction of whether or not it is justified to undertake further clinical testing which until recent times has been normally done by prostate biopsy. At present, for the initial work-up (for example by the primary care physician in the UK), the decision to refer a patient for a biopsy is based entirely on age-specific normal ranges for PSA, which thus fails to take into account other risk factors (which may either increase or decrease underlying risk of significant prostate cancer). Transrectal ultrasound (TRUS) biopsy is then normally undertaken to assess whether histologically confirmed cancer is present. More recently, MRI has been looked at, either to guide the biopsy procedure or as a diagnostic modality in its own right.

Risk prediction models allow for the integration of multiple factors and with increasing markers of disease and of treatment response becoming available it is likely that risk-based models will be further developed and utilised and will continue to gain wider clinical utility and significance. A number of issues remain to be fully assessed though, and there remains particular debate as to how best to define clinically significant disease. This uncertainty adds to the difficulty in developing risk prediction models and in obtaining wide spread clinical usage.

There has been a growing number of prostate cancer risk prediction models developed over recent years aimed at helping the clinician and or the man himself estimate their chance of having prostate cancer either now or in a given future period (e.g. 10 years, lifetime, etc.). The large numbers of risk 
calculators for different periods of risk has to some extent led to confusion and overload. It is not clear how effective many of these risk predictions might be when employed at the first line medical health check as many were developed in the urology clinic setting. Furthermore, there is very little knowledge or use of these risk calculators by many frontline physicians.

Risk calculators that have potential application in the community setting need to be based on factors and tests that can be readily assessed in the initial diagnostic setting. Among the first of these to gain prominence in Canada and the USA was the Sunnybrook risk calculator which was constructed using all known risk factors to assess an individual's risk of having prostate cancer for men undergoing a PSA test [1]. It was derived from a study of 3108 Canadian men who underwent a prostate biopsy, 408 of whom had a normal PSA level. Overall, $42 \%$ were diagnosed with prostate cancer, $11 \%$ of which were high grade (Gleason grades 7, 8, 9 and 10). These results were used to construct a nomogram to predict the presence of both "any prostate cancer" and the aggressive form of prostate cancer. Factors included in the model were age, family history of prostate cancer, ethnicity, urinary symptoms (AUA symptom score), PSA, PSA free-to-total ratio and DRE. The area under the receiver-operator curve (AUC) (prediction for presence of prostate cancer) was 0.74 ( $95 \%$ confidence interval (C.I.) 0.71-0.81) and AUC for high-grade cancer was 0.77 (95\% C.I. 0.74-0.81). Internal validation was carried out by comparing the nomogram with conventional screening using only PSA and digital rectal examination (DRE). The AUC for conventional PCA detection was significantly lower $(0.62,95 \%$ C.I. $0.58-0.66$ for the presence of prostate cancer and $0.69,95 \%$ C.I. $0.65-0.72$ for the presence of high-grade cancer). The risk prediction model was wellcalibrated, yielding estimates of risk similar to the prevalence at biopsy [1]. Extensions to the original model in terms of evaluating genetic markers in the form of selected single nucleotide polymorphism did not add to the clinical utility of the approach [2]. The calculator has been principally used at selected centres in Canada and the USA as a guide to aid clinical decision-making.

A further leading community-based calculator was developed in the USA using data from the Prostate Cancer Prevention Trial (PCPT). The first version of the PCPT prostate cancer risk calculator (PCPTRC) was published in 2006 [3]. The study used logistic regression to produce a model which includes age at biopsy, race, family history of prostate cancer, PSA level, PSA velocity, DRE result and previous prostate biopsy results. The PCPTRC can be used in settings where regular PSA testing is employed such as the USA.

The PCPTRC model has an average AUC for the prediction of prostate cancer of 0.70 with a standard deviation= 0.006 [3]. It was externally validated in the San Antonio Centre of Biomarkers of Risk for Prostate Cancer (SABOR) cohort of the Early Detection Research Network which was a younger and more ethnically diverse population than the original PCPT trial population and had an AUC of 0.66 [4]. In 2010, this nomogram was further externally validated in a Portuguese population with an AUC of 0.70 (Oliveira et al., 2010).

For comparison, Nam et al. conducted an external validation of their Sunnybrook nomogram-based prostate cancer risk calculator (SRC) and compared with the PCPTRC in a prospective, multi-institutional study of 2130 patients who underwent a prostate biopsy for prostate cancer detection. The AUC for the SRC was 0.67 (95\% CI, 0.65 to 0.69 ), and the AUC for the PCPTRC was 0.61 (95\% CI, 0.59 to 0.64). The AUC was higher for predicting aggressive disease from the SRC $(0.72 ; 95 \% \mathrm{CI}, 0.70$ to 0.75$)$ compared with that from the PCPRT $(0.67 ; 95 \%$ CI, 0.64 to 0.70$)$ [5].

The PCPTRC was also validated in ten international biopsy cohorts, and the results suggested variations in the AUCs between cohorts. The authors concluded that the variations were due to different criteria for work-up before biopsy within these cohorts [6].

In 2014, a further version of the PCPTRC (Version 2.0) was published [7]. The newer model aims to predict the risk of no vs low-grade (Gleason grade $<7$ ) vs high-grade cancer (Gleason grade 7). In this current model, the authors applied percent free-PSA and did not use PSA velocity. The parameters included in the model are therefore PSA, digital rectal examination, age, race, prior biopsy and family history. The new PCPTRC 2.0 either with or without percent free-PSA is claimed to be well-validated externally. However, the inclusion of percent free, or free/total PSA may limit applicability in the initial diagnostic setting as this test may not be routinely available in many settings.

In Europe, the Prostate Cancer Risk Calculator (PCRC) was constructed using data from Rotterdam, one of the study centres involved in the European Randomised Study of Prostate Cancer Screening (ERSPC). The calculator is designed to avoid unnecessary biopsies and to increase detection of potentially aggressive prostate cancer $[8,9]$. The ERSPC Prostate Cancer Risk Calculator was based on 6288 Dutch men and utilised their biopsy results to construct a graphic device which uses six steps to estimate risk. The study reported an AUC up to step 3 of 0.77 (95\% C.I. 0.74-0.79) [10]. The risk calculator (up to step 3) was externally validated with 390 Portuguese patients who had ten-core systematic transrectal prostate biopsies; it had a high predictive accuracy of $77.9 \%$ [11].

The Rotterdam group of the ERSPC trial has subsequently developed a modification of the risk calculator to allow for the inclusion of estimated prostate volume by DRE (ERSPC3+ DRE). Previously, prostate volume was obtained by ultrasound of the prostate gland which limited the practicability of the risk calculator in a UK primary care setting for example. 
The modified ERSPC risk calculator therefore offers a good tool for use in primary care, although its performance in comparison to the Sunnybrook calculator remains to be established. Even with a relatively rough estimation of the total prostate volume at DRE $(25,40$ or $60 \mathrm{cc})$, the adapted ERSPC Risk calculator performs better than PSA alone and PSA in combination with DRE [12].

The European Randomised Study of Screening for Prostate Cancer Risk Calculator (ERSPC RC) has also been updated to predict potentially aggressive prostate cancer, defined as Gleason $\geq 7$ and/or T-stage $>$ T2B. The further version of the calculator showed an AUC of 0.86 (95\% CI 0.84-0.86) on the ERSPC dataset (AUC PSA alone 0.74). AUC's of the ERSPC $\mathrm{RC}$ and the PCPT RC for high-grade PC on a Canadian $(N=$ 982, clinical cohort) validation cohort were $0.76(0.72-0.79)$ and $0.68(0.64-0.72), p<0.0001$, respectively. The AUC of PSA alone was $0.61[11,13]$.

A recent publication has looked specifically at a head to head comparison of prostate cancer risk prediction models [14••]. The meta-analysis used three main criteria including the presence of PSA level in the model, models that have been validated in $\geq 5$ study populations and models that have assessed area under the curve (AUC) for prediction of any or clinically significant PCA. The authors identified 6 out of 127 prostate cancer prediction models met the criteria. These risk prediction models were: Prostataclass, Finne, Karakiewcz, Prostate Cancer Prevention Trial (PCPT), Chun and the European Randomised Study of Screening for Prostate Cancer Risk Calculator 3 (ERSPC RC3). The ERSPC RC3 and Prostataclass had the highest AUC (0.79). The PCPT did not show any difference in its ability to predict any prostate cancer compared to PSA testing alone (AUC $=0.66$ for both), but it did have better ability to predict clinically significant prostate cancer ( $\mathrm{AUC}=0.71$ and $\mathrm{AUC}=0.66$, respectively). Five of these risk predictions except by Chun have incorporated DRE into the model. Chun instead used sampling TRUS from biopsy. Calibration measures of the models were in general poorly reported. Only Finne and Chun have described calibration of the risk algorithm fully [14••]. The conclusion of the meta-analysis was that risk prediction models do perform better than PSA alone.

In addition to the highlighted community-based risk calculators above, there are over a hundred further calculators available which calculate a variety of risks principally for the clinical situation $[15,16]$.

Approaches to get widespread uptake of any of the calculators in primary care/front line diagnostics remain to be done, and the risk calculators have to be further improved to better detect clinically significant cancers.

Other issues still remain to be evaluated in that costs and cost effectiveness become keys as the diagnostic approach has to be used in large numbers of men. Furthermore, newly emerging risk prediction approaches include specific high and low penetrance genes (rather than questionnaire based on family history) which may affect men's consideration of their use as they have potential implications for other family members.

The issue of how best to further monitor men after initial risk assessment has been done also remains to be established.

\section{The Future of Diagnostic Risk Assessment}

As many of the above calculators offer good potential to aid in the diagnostic pathway for PCA, the choice and use of which calculator needs to be determined by the clinical setting in which it is to be used and the wider full head to head comparisons can often be difficult to evaluate. There remains an important issue in terms of the error inherent in defining the gold standard of diagnosis, i.e. all the calculators above relied on the TRUS biopsy as being definitive but this is known to have error rates as high as $30 \%$. Hence, the new paradigm is how these calculators will perform in an optimally investigated population (e.g. using MRI and targeted biopsies to define whether cancer is truly present).

Perhaps leading the way in terms of evaluating the use of risk calculators in a near population-based community setting, recent work in Sweden has been undertaken to investigate the clinical advantages of using a combined risk assessment to reduce the over diagnosis of low-grade tumours and the study results are expected to be published soon. It is likely that the study will be able to demonstrate in a setting of wide-scale population-based "screening" the sorts of advantages seen in previous more constrained clinic-based evaluations. The Swedish research team are looking to continue their evaluations to include advances in biopsy procedures including MRI guided biopsies.

In addition, there are a number of further ongoing programs of work evaluating the practical issues involved in changing the diagnostic pathways and to further develop the risk models used in practice. Examples of such programs include the "Riskman" program in the UK which is underway to develop and evaluate the "Riskman" calculator, a risk assessment algorithm for GPs to assess which men should be referred for prostate biopsy [17]. The calculator is being developed for use in the primary care setting in the UK. It will be constructed using the most informative markers of existing communitybased calculators together with the best combination of further biomarkers, epidemiological factors and SNPs now available, for potential incorporation to produce a calculator with optimised performance and costs for future wide-scale use in the NHS in the UK. The program is focused not only on further developing the risk prediction models but also on the best translational routes to direct implementation in the NHS.

Future risk calculators will need to further evaluate a range of new and emerging biomarkers both in terms of genetic 
markers, blood-based proteins and potentially also informative urine markers (see Table 1).

The role of genetic markers also needs further consideration, particularly in terms of individual risk prediction versus population stratification. To date, the rapid gene sequencing advanced by the Human Genome Project and genome-wide association studies have identified over 100 single nucleotide polymorphisms that predict PCA. In the UK, the PROFILE study by Eeles et al. is utilising a risk score based on a combined SNPs profile to identify a population of men at high risk of developing prostate cancer. At present, such tests are likely to be offered through genetic counselling clinics, but in the future, direct-to-consumer (DTC) genetic testing makes it likely that such tests will be commercially marketed directly to the public. Men using DTC's testing in this way would not have direct access to a doctor or genetic counsellor's guidance, creating possible anxiety or false reassurance pushing people to make medical decisions on incomplete or misunderstood information [18].

Alternatively, utilising such genetic markers has the potential to be used either to add to existing risk prediction models or to stratify the population by predisposition risk and then employ conventional risk prediction tools.

Issues of risk communication however remain pervasive in the use of risk prediction models. The wider issue of cancer risk communication is not unique to prostate cancer risk calculators. Unresolved at this time are how to best present the information from a risk model and what the patient understands by the outputs. Thus, greater future research should also focus on risk presentation and risk

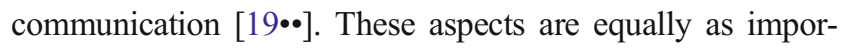
tant as building reliable risk calculators. Health professionals need to understand how best to communicate the risk and publics views and need to be able to understand the meaning of risk and potential ways to manage their risk profile. Colditz and Wei (in the parallel paper in this series) argue, as does Moons [20, 21], that the end user must be considered from the first steps in model development to assure transfer to use that will improve health outcomes.

Han et al. investigated patient experience with prostate cancer with risk information and their perceptions of the value of personalised risk information using a focus group approach. They reported most prostate cancer patients had very little experience in utilising risk information. Some patients perceived personalised risk information as less valuable than other types of evidence [22]. How best to communicate and inform the results thus remains to be established.

In sum, risk-based prostate cancer detection in the community offers potentially significant improvements in performance over the current PSA threshold-based approach particularly as a first-line diagnostic testing approach. Nearly all the leading community-based risk calculators, however, still require an assessment of the prostate by digital rectal examination (DRE). This requirement limits their widest possible use, particularly in some ethnic groups and some clinical settings. Further work on improving the accuracy and removing the need for undertaking a DRE assessment would widen still further their utility and likely benefit to improving the diagnostic pathway for PCA. How best to communicate the results, how to create awareness of them and how to use them in the clinical setting remain key issues to be resolved.

\section{Management Options}

Once diagnosed and clinically characterised, there is a need to correctly risk stratify those patients that need the most effective treatment be it surgery, radiotherapy, chemotherapy or combinations of any of these. At present, the only robust choices on treatment allocation is the choice between AS and radical therapy, although there is a lot of research effort
Table 1 Emerging biomarkers for characterising the risk to the individual from their particular cancer

\begin{tabular}{ll}
\hline Category & Biomarker \\
\hline Potential diagnostic & K4 score \\
& Progensa PCA3 Assay3 \\
& PSA isoform p2PSA and PHI \\
& $\beta$-microseminoprotein MSMB (PSP94) \\
& Macrophage inhibitory cytokine 1 (MIC-1 or GDF-15) \\
& Genetic marker panel i.e., OncotypeDX \\
& EN2 (urine) \\
& TMPRSS2-ERG \\
& ConfirmMDx \\
Risk stratification for defining tumour & Promark ${ }^{\mathrm{TM}}$ \\
characteristics and treatment choice & Decipher \\
& "Know Error" \\
& Prolaris
\end{tabular}


to more precisely risk assess allow more individualised treatment approaches.

To date, clinicians typically have been using different forms of risk stratification particularly to help with the management of localised and locally advanced non-metastatic prostate cancer. The easiest and most commonly used method of risk stratification is that of D'Amico et al. [23]. Three combinations of parameters, including preoperative PSA, clinical stage and Gleason score which are used to classify patients diagnosed with prostate cancer into low-, intermediate-, and high-risk categories.

In 2012, a critical review by Rodrigues et al. offered evidence that this simplistic model needed modification based on clinical prognostic evidence. The proposed changes included the creation of new risk group for "very-low" risk patients. Further stratification based on the splitting of intermediaterisk into a low- and high-intermediate-risk groups and the clarification of the interface between intermediate- and highrisk disease was desirable [24]. For future enhancements, the authors also recommended the potential use of other novel pathological factors such as percentage of biopsy core positive and subtype of Gleason grade 7.

By comparison, the Cancer of the Prostate Risk Assessment (UCSF-CAPRA) score was invented by incorporating two further factors including age at diagnosis and percentage of positive biopsy core. The CAPRA score ranges from 0 to 10 score with score of 0 to 2 indicative low-risk, score of 3 to 5 indicative intermediate-risk and score of 6 to 10 indicative of high risk [25].

At present, prostate cancer risk stratification mainly relies on clinical stage, initial PSA level, and Gleason score with some risk stratification scheme adding on further prognostic factors such as percentage of positive biopsy cores, PSA doubling time, PSA velocity, etc.

Future risk stratification will incorporate a much wider range of biomarkers. Current promising treatment stratification markers are shown in Table 1, but these are likely to be added to including epigenetic, microRNA and even circulated tumour cells themselves. These should provide better ways to characterise the individualised patient's disease risk and hence appropriate management can be provided and are part of the big drive to "personalised medicine".

In summary, at the present time, given that the best ways to treat different forms of PCA are not yet fully established has led to some confusion on how to best risk stratify men with diagnosed cancers. Ongoing large scale clinical evaluations of the best way to treat the various forms of prostate cancer, including for example the UK-based ProtecT trial will provide opportunities to further develop improved risk prediction to improve the prediction of best treatment options to aid both patients and clinicians alike. Furthermore, the very recent the International Society of Urological Pathology (ISUP) grading prognostic factors which will likely change all the current risk criteria [26].

\section{Conclusions}

Risk offers a unifying approach to both the diagnosis and risk stratification of men both before and after a diagnosis of PCA. Several areas of further development are required and these could best be done in an incremental way whilst starting to use the approach in practice.

Challenges remain including how best to use the individual risk calculators in the context of population stratification. There remains a lack of large scale studies on the actual usage/translation into practice and a number of factors work against this, i.e. who decides which is the best one and how to get widespread uptake and acceptance and how best to evaluate them (including choosing appropriate levels of AUC, calibration and validity). Relative costs will come into play depending on where they are going to be employed. Many risk prediction models derived from one country/healthcare system may not be accepted in other countries. Furthermore, what do men themselves feel about risk-qualitative studies suggests they often decide on particular actions based more in a heuristic (rule of thumb) way. The whole issue of communication of the risk is very important; we are not all the same and whilst some men might think one risk is high whilst others may consider differently. It is further worth noting, different attitudes within ethnic groups to risk, i.e. black men in UK were less likely to act at a given risk than white men [27]. In addition, other co-morbidities must feed into the consideration and is the decision on appropriate further action to be taken made by the medical profession or by the man himself.

Finally, how best can such estimates of PCA risk be used alongside estimates for other cancers/serious conditions which are also rapidly being developed.

In sum, risk prediction for PCA does improve particularly the diagnostic pathway for PCA and has the potential to be further developed to enhance risk stratification to help define better disease management. How best to use existing models in practice and to further enhance them remains to be fully established. If such translational studies are set up, future risk prediction improvements could be further refined in practice and in an ongoing way to more rapidly realise the full potential that the approach offers.

\section{Compliance with Ethics Guidelines}

Conflict of Interest Kenneth Ross Muir, Artitaya Lophatananon, Vincent Gnanapragasam and Jonathan Rees declare that they have no conflict of interest. 
Human and Animal Rights and Informed Consent This article does not contain any studies with human or animal subjects performed by any of the authors.

\section{References}

Papers of particular interest, published recently, have been highlighted as:

• Of major importance

1. Nam RK, Toi A, Klotz LH, Trachtenberg J, Jewett MA, Appu S, et al. Assessing individual risk for prostate cancer. J Clin Oncol. 2007;25(24):3582-8.

2. Nam RK, Zhang WW, Trachtenberg J, Seth A, Klotz LH, Stanimirovic A, et al. Utility of incorporating genetic variants for the early detection of prostate cancer. Clin Cancer Res. 2009;15(5): 1787-93.

3. Thompson IM, Ankerst DP, Chi C, Goodman PJ, Tangen CM, Lucia MS, et al. Assessing prostate cancer risk: results from the prostate cancer prevention trial. J Natl Cancer Inst. 2006;98(8): 529-34.

4. Parekh DJ, Ankerst DP, Higgins BA, Hernandez J, Canby-Hagino E, Brand T, et al. External validation of the prostate cancer prevention trial risk calculator in a screened population. Urology. 2006;68(6):1152-5.

5. Nam RK, Kattan MW, Chin JL, Trachtenberg J, Singal R, Rendon $\mathrm{R}$, et al. Prospective multi-institutional study evaluating the performance of prostate cancer risk calculators. J Clin Oncol. 2011;29(22):2959-64.

6. Ankerst DP, Boeck A, Freedland SJ, Thompson IM, Cronin AM, Roobol MJ, et al. Evaluating the PCPT risk calculator in ten international biopsy cohorts: results from the prostate biopsy collaborative group. World J Urol. 2012;30(2):181-7.

7. Ankerst DP, Hoefler J, Bock S, Goodman PJ, Vickers A, Hernandez $\mathrm{J}$, et al. Prostate cancer prevention trial risk calculator 2.0 for the prediction of low- vs high-grade prostate cancer. Urology. 2014;83(6):1362-7.

8. Kranse R, Roobol M, Schroder FH. A graphical device to represent the outcomes of a logistic regression analysis. Prostate. 2008;68(15):1674-80.

9. Steyerberg EW, Roobol MJ, Kattan MW, van der Kwast TH, de Koning HJ, Schroder FH. Prediction of indolent prostate cancer: validation and updating of a prognostic nomogram. J Urol. 2007;177(1):107-12. discussion 12.

10. Serag H, Banerjee S, Saeb-Parsy K, Irving S, Wright K, Stearn S, et al. Risk profiles of prostate cancers identified from UK primary care using national referral guidelines. Br J Cancer. 2012;106(3): 436-9.

11. Roobol M, Van Vugt H, Kranse R, Van Leeuwen P, Bangma C. Validation of the ERSPC risk calculator in a clinical setting. European Association of Urology Annual Congress Vienna, Austria.

12. Roobol MJ, van Vugt HA, Loeb S, Zhu X, Bul M, Bangma CH, et al. Prediction of prostate cancer risk: the role of prostate volume and digital rectal examination in the ERSPC risk calculators. Eur Urol. 2012;61(3):577-83.
13. Trottier G, Roobol MJ, Lawrentschuk N, Bostrom PJ, Fernandes KA, Finelli A, et al. Comparison of risk calculators from the prostate cancer prevention trial and the european randomized study of screening for prostate cancer in a contemporary Canadian cohort. BJU Int. 2011;108(8 Pt 2):E237-44.

14.• Louie KS, Seigneurin A, Cathcart P, Sasieni P. Do prostate cancer risk models improve the predictive accuracy of PSA screening? A meta-analysis. Ann Oncol. 2015;26(5):848-64. Important overview and comparison some of the leading risk calculators.

15. Shariat SF, Karakiewicz PI, Suardi N, Kattan MW. Comparison of nomograms with other methods for predicting outcomes in prostate cancer: a critical analysis of the literature. Clin Cancer Res. 2008;14(14):4400-7.

16. Shariat SF, Kattan MW, Vickers AJ, Karakiewicz PI, Scardino PT. Critical review of prostate cancer predictive tools. Future Oncol. 2009;5(10):1555-84.

17. University of Manchester. New approach to prostate cancer screening needed. [Internet] Manchester: http://www.manchester.ac.uk/ discover/news/article/?id=11719; 2014 [2015]; Available from: http://www.manchester.ac.uk/discover/news/article/?id=11719.

18. Institute of National Cancer. Genetic Testing for Hereditary Cancer Syndromes. [Internet]: http://www.cancer.gov/cancertopics/ factsheet/Risk/genetic-testing.; 2013 [2015]; Available from: http://www.cancer.gov/cancertopics/factsheet/Risk/genetic-testing.

19.• Weinstein ND, Atwood K, Puleo E, Fletcher R, Colditz G, Emmons KM. Colon cancer: risk perceptions and risk communication. J Health Commun. 2004;9(1):53-65. 65. Paper discussing the wider issue around risk communication.

20. Moons KG, Kengne AP, Grobbee DE, Royston P, Vergouwe Y, Altman DG, et al. Risk prediction models: II. External validation, model updating, and impact assessment. Heart. 2012;98(9):691-8. Heart. [Research Support, Non-U.S. Gov't Review].

21. Moons KG, Kengne AP, Woodward M, Royston P, Vergouwe Y, Altman DG, et al. Risk prediction models: I. Development, internal validation, and assessing the incremental value of a new (bio)marker. Heart. 2012;98(9):683-90 [Research Support, Non-U.S. Gov’t Review].

22. Han PK, Hootsmans N, Neilson M, Roy B, Kungel T, Gutheil C, et al. The value of personalised risk information: a qualitative study of the perceptions of patients with prostate cancer. BMJ Open. 2013;3(9):e003226.

23. D'Amico AV, Whittington R, Malkowicz S, et al. Blochemical outcome after radical prostatectomy, external beam radiation therapy, or interstitial radiation therapy for clinically localized prostate cancer. JAMA. 1998;280(11):969-74.

24. Rodrigues G, Warde P, Pickles T, Crook J, Brundage M, Souhami $\mathrm{L}$, et al. Pre-treatment risk stratification of prostate cancer patients: a critical review. Can Urol Assoc J. 2012;6(2):121-7.

25. Cooperberg MR, Broering JM, Carroll PR. Risk assessment for prostate cancer metastasis and mortality at the time of diagnosis. $\mathrm{J}$ Natl Cancer Inst. 2009;101(12):878-87.

26. Delahunt B, Egevad L, Srigley JR, Steigler A, Murray JD, Atkinson $\mathrm{C}$, et al. Validation of international society of urological pathology (ISUP) grading for prostatic adenocarcinoma in thin core biopsies using TROG 03.04 'RADAR' trial clinical data. Pathology. 2015;47(6):520-5.

27. Martins T, Ukoumunne OC, Banks J, Raine R, Hamilton W. Ethnic differences in patients' preferences for prostate cancer investigation: a vignette-based survey in primary care. Br J Gen Pract. 2015;65(632):e161-70. doi:10.3399/bjgp15X683965. 